Asian Pacific Journal of Reproduction

Journal homepage: www.apjr.net

\title{
Pregnancy outcome of day 3 versus day 5 embryo transfer: A retrospective analysis
}

\author{
Samaher Alfaraj ${ }^{1 \otimes}$, Fatima Alzaher ${ }^{2}$, Sahar Alshwaiaer ${ }^{1}$, Anwar Ahmed ${ }^{3}$ \\ ${ }^{l}$ Department of Obstetrics and Gynecology, Reproductive Endocrinology and Infertility Unit, King Abdul-Aziz Medical City, Riyadh, Saudi Arabia \\ ${ }^{2}$ Department of Obstetrics and Gynecology, Ministry of Health, Dammam, Saudi Arabia \\ ${ }^{3}$ Assistant Professor of Biostatistics, Department of Epidemiology and Biostatistics College of Public Health and Health Informatics King Saud Bin \\ Abdulaziz University for Health Sciences National Guard Health Affairs Riyadh, KSA
}

\section{ARTICLE INFO}

Article history:

Received 5 December 2016

Revision 15 December 2016

Accepted 10 January 2017

Available online 1 March 2017

\section{Keywords:}

Embryo transfer

Blastocyst

Early cleavage stage

Pregnancy outcomes

Sequential culture media

\begin{abstract}
Objective: To compare the pregnancy rate between day 3 and day 5 transfer regardless grades of embryos and number of transferred embryo. Methods: Retrospective cohort, a total of seven hundred and four patients met our inclusion criteria, with 411 had day 3 embryo transfer and 293 had day 5 embryo transfer. The patients who were older than 40 years old were excluded. Embryo transfer was carried out in all patients in both transfer groups. Results: Both clinical pregnancy rate and implantation rate did not show any statistically significant difference between the day 3 and day five transfer groups. These were $44 \%$ vs. $45 \%$ with $P=0.82$ and $19 \%$ vs. $19 \%$ with $P=0.99$ respectively. An increase of miscarriage rate with day 5 embryo transferred compare with day $3(12.0 \%$ vs. $4.4 \%, P=0.01)$, but no significant difference was found about biochemical pregnancy rate $(P=0.52)$. Conclusions: Transferring embryo at day 5 may not provide any additional benefit over day 3 transfers to patients. In addition, it increases the risk of miscarriage. Further studies of this issue needed for confirming our findings.
\end{abstract}

\section{Introduction}

Infertility is a common condition with important psychological, economic, demographic and medical implications. Assisted reproductive technology can alleviate the burden of infertility on individuals and families, but it can also present challenges to health care providers as evidenced by the low implantation and pregnancy rate.

Several options have been proposed to increase the in-vitro fertilization (IVF) success rate, one of them is the day of embryo transfer. Human embryos obtained through in-vitro techniques are routinely transferred to the uterus on day 2 or 3 when they are at the 4-8 cell stage. The implantation rate of these embryos is disappointingly low and range between $10 \%-20 \%$. Therefore, it has been suggested that selecting transferring embryos at the blastocyst stage instead of at an earlier stage without knowing their developmental capability might enhance the implantation rate[1].

Development of sequential tissue culture media attempts to give the growing embryo its metabolic and chemical needs, which has resulted in an extension of the embryo culture period to the blastocyst stage[2-5]. Therefore, the transfer of human blastocysts in
IVF should result in an increased implantation rate and enable the transfer of fewer, but higher quality embryos. This would maintain the high pregnancy rate while controlling the incidence of highorder multiple gestations[5-8].

During the last decade, the extension of embryo culture to day 5 has gained popularity in IVF practice. The main reasons for this new strategy are mainly scientific. First, the embryo does not implant before day 5. Second, the understanding of the different metabolic needs of the embryo during its development and the evolution of new sequential media render that possible the culture of embryos in vitro until the day 5 with relatively good survival rates[9].

Recent reports in literature showed increased pregnancy rate with blastocyst transfer[10]. According to Ronal et al.[11] blastocyst transfer resulted in fewer embryos transferred, with a trend toward improving clinical pregnancy and twin pregnancy rate. The study of Peter et al.[2] concluded that blastocyst transfer may lead to a higher pregnancy rate with an overall better take -home baby rate.

Evangelos et al.[9] reached a conclusion in a systematic review

This is an open access article distributed under the terms of the Creative Commons Attribution-Non Commercial-Share Alike 3.0 License, which allows others to remix, tweak and buid upon the work non-commercially, as long as the author is credited and the new creations are licensed under the identical terms.

For reprints contact: reprints@medknow.com

C2017 Asian Pacific Journal of Reproduction Produced by Wolters Kluwer- Medknow

How to cite this article: Samaher Alfaraj, Fatima Alzaher, Sahar Alshwaiaer, Anwar Ahmed. Pregnancy outcome of day 3 versus day 5 embryo transfer: A retrospective analysis. Asian Pac J Reprod 2017; 6(2): 89-92. 
and meta-analysis that the probability of live birth after fresh IVF was significantly higher after blastocyst-stage embryo transfer as compared to cleavage-stage embryo transfer when equal number of embryos were transferred in the two groups compared. A randomized prospective study Evangelos et al.[9] investigated the threshold number of embryos available on day 3 of embryo culture and live birth rate among day 3 embryo transfer and day 5 embryo transfer. The study showed that a threshold of four good embryos on day 3 of embryo culture appears to indicate that the patient would benefit from embryo transfer at the blastocyst stage and have a better chance of achieving a live delivery than embryo transfer at cleavagestage[12].

In contrast, Serdar et al.[1] in controlled randomized trial showed day 3 and day 5 transfer had similar pregnancy, implantation and twinning rates. Day 5 transfers had no advantages over day 3 transfers. Howard et al.[13] did a retrospective comparison of day 5 blastocyst transfer versus day 3 embryo transfer. In their program, they were equally successful with blastocyst transfer and day 3 transfer, with the exception of patients with few oocytes who benefited from day 3 transfer, and patients over age 39 with a good ovarian response to gonadotropins who benefited from blastocyst transfer.

Assisted reproduction programs still lack high implantation rates. Whether blastocyst-stage transfer offers any real benefit to infertile couples is still controversial[12]. The aim of our study is to compare the pregnancy rate between day 3 and day 5 transfer regardless grades of embryos and number of transferred embryo.

\section{Materials and methods}

\subsection{Study setting, design, and participants}

A retrospective cohort study was undertaken at King Abdul-Aziz Medical City and King Fahd National Guard Hospital, Department of Obstetrics and Gynecology, Reproductive Endocrinology and Infertility Unit. Ethical approval was obtained from King Abdullah Research center and an earlier consent was obtained from all couples to share their information for research purpose.

\subsection{Ethical approval}

This study was approved by the Research and Ethics Committee at the College of Public Health and Health Informatics, King Saud Bin Abdulaziz University for Health Sciences, Riyadh, Saudi Arabia. Informed consent was obtained from all participants who agreed to share the outcomes of their cycles for research purposes.

\subsection{Study selection criteria}

Cohort of IVF/intracytoplasmic sperm injection (ICSI) cycles performed day 3 and day 5 embryo transfers included all patients who underwent IVF or ICSI in our unit during January 2014 to April 2015 and female age $\leqslant 40$ years old. We excluded all frozen sperms cycle.

\subsection{IVF cycle protocol at KAMC}

\subsubsection{Ovarian stimulation}

The patients who are included in the study were managed as per the following protocol: The short GnRH-antagonist protocol is one of the protocols used in our unit. Controlled ovarian stimulation started on the day 2 to day 4 of menstrual cycle either by recombinant follicle-stimulating hormone (Gonal f), (Bravelle), or human menopausal gonadotrophins (menogon or merional) for $7 \mathrm{~d}$ if baseline endometrial thickness $<6 \mathrm{~mm}$ and ovaries are normal. Starting injection of GnRH-antagonist (cetrorelix acetate $0.25 \mathrm{mg}$ ) SC at day 5 of stimulation carried out. On day 8, estradiol level and vaginal ultrasonography will be repeated and the dose of gonadotrophins continued or adjusted according to these parameters. If there are two mature follicles more $\geqslant 18 \mathrm{~mm}$, patient will be booked for ovum pick up procedure and prescribed injection HCG $10000 \mathrm{IU}$. She will be scheduled for ovum pick up after $36 \mathrm{~h}$ from human chorionic gonadotropin (HCG) injection. The procedure will be performed after taking written consent from the patient. It will be carried under moderate sedation with maximum dose $1.5 \mathrm{mcg} / \mathrm{kg}$ of intravenous midazolam $2 \mathrm{mg}$ and fentanyl $0.5 \mathrm{mcg} / \mathrm{kg}$. Then the patient will be discharged home if she is stable. Husband will give semen sample in the same day of ovum pick up and the cycles either will be for IVF or ICSI.

Oocytes will be incubated in fertilization medium and this culture will be carried out in 4-well multi-dishes containing each $40 \mathrm{~mL}$ micodrops under mineral oil (for ICSI cases) or in $800 \mathrm{~mL}$ of sequential media (for IVF cases) at $37{ }^{\circ} \mathrm{C}$ in a humidified atmosphere at $6 \% \mathrm{CO}_{2}$ in air. Sixteen to twenty hours after insemination or ICSI, the oocytes will be assessed for the presence of two pronuclei and after rinsing they will be transferred into cleavage medium for an additional period of $48 \mathrm{~h}$. Extension of culture to day 5 will start after this step. After that, the embryos will be placed in wells or microdrops of blastocyst medium on day 3 and further culture for 48 $\mathrm{h}$ to reach blastocyst stage.

\subsubsection{Embryo transfer procedure}

Embryo transfer procedure carried out either on day 3 or day 5 of ovum pickup procedure. Supplementary progesterone (cyclogest) started after $2 \mathrm{~d}$ of ovum pick up and may last for $12 \mathrm{wk}$ if pregnancy test is positive, otherwise till the day of pregnancy test if it is negative.

\subsection{Operational definitions}

Our primary outcome measure is the clinical pregnancy rate and it is a positive serum pregnancy test estimated by $\beta$-HCG in blood $>5 \mathrm{mIU}$ with trans abdominal ultrasound finding a gestational sac. The secondary outcomes are the fertilization rate which is defined as number of oocyte injected divided by the number of embryos fertilized and the implantation rate is defined as number of gestational sac seen in ultrasound divided by number of transfer embryos.

\subsection{IVF outcome assessment}

A compression between day 3 and day 5 embryo transferred were carried out in relation of pregnancy rate implantation rate, fertilization rate and miscarriage rate. 


\subsection{Data management and analysis plan}

Data were analysed to compare between day 3 and day 5 embryo transferred using the statistical package $\left(\operatorname{SPSS}^{\oplus}\right)$ version 22.0 (Chicago, Illinois, USA) at a significance level $(P<0.05)$ while age is presented as mean $\pm \mathrm{SD}$.

\section{Results}

Seven hundred and four patients met our inclusion criteria, with 411 patients having day 3 embryo transfer and 293 patients having day 5 embryo transfer. The patients who were older than 40 years old were excluded from this study. Embryo transfer was carried out in all patients in both transfer groups. Statistical analysis of the data revealed no significant differences in age between the day 3 and day 5 transfer groups. Embryos transferred on day 3 was $(31.4 \pm 4.5)$ and on day 5 was $(31.3 \pm 4.5)$ with $P=0.529$ (Table 1$)$.

Table 1

Patient demographics and characteristics.

\begin{tabular}{cccccc}
\hline \multicolumn{1}{c}{ Indexes } & & Overall & Day 3 & Day 5 & $P$ \\
\hline $\begin{array}{c}\text { Number of } \\
\text { patients } \\
\text { Age of }\end{array}$ & - & 704 & 411 & 293 & - \\
female (20- & - & $31.4 \pm 4.5$ & $31.3 \pm 4.5$ & $31.3 \pm 4.5$ & 0.529 \\
$40)$ & & & & & \\
Types of & Primary & $307(44.0)$ & $187(46.2)$ & $120(41.0)$ & 0.171 \\
infertility & Secibdary & $391(56.0)$ & $218(53.8)$ & $173(59.0)$ & - \\
& Female & $248(35.5)$ & $148(36.5)$ & $100(34.1)$ & - \\
Causes of & Male & $251(36.0)$ & $152(37.5)$ & $99(33.8)$ & \\
infertility & Combined & $129(18.5)$ & $68(16.8)$ & $61(20.8)$ & 0.362 \\
\hline
\end{tabular}

"Data are expressed as $n(\%)$.

There was no difference between day 3 group and day 5 group regarding the infertility causes: male factor $(n=251)$ and female factor $(n=248)$, combined $(n=129)$ idiopathic $(n=70)$ with $P=0.362$.

The type of infertility for the two transfer groups were comparable (primary $v s$. secondary infertility) $P=0.17$, as well as the type of assisted reproductive technology (IVF vs. ICSI) $P=0.42$. Table 2 shows details of pregnancy outcomes and comparisons between two groups. Both clinical pregnancy rate and implantation rate did not show any statistically significant difference between the day 3 and day 5 transfer groups. Pregnancy rate respectively were $44 \%$ vs. $45 \%$ with $P=0.82$ and $19 \%$ vs. $19 \%$ with $P=0.99$ respectively.

Table 2

Pregnancy outcome.

\begin{tabular}{lccc}
\hline \multicolumn{1}{c}{ Indexes } & Day 3 & Day 5 & $P$ \\
\hline Clinical pregnancy rate & $182 / 411(44.30 \%)$ & $133 / 293(45.40 \%)$ & 0.818 \\
Implantation rate & $178 / 919(19.40 \%)$ & $116 / 598(19.40 \%)$ & 0.992 \\
Miscarriage rate & $8 / 182(4.40 \%)$ & $16 / 133(12.03 \%)$ & $0.011^{*}$ \\
Biochemical pregnancy rate & $8 / 182(4.40 \%)$ & $4 / 133(3.00 \%)$ & 0.522 \\
Multiple pregnancy rate & $45 / 182(24.70 \%)$ & $28 / 133(21.10 \%)$ & 0.440 \\
\hline
\end{tabular}

"The result is significant at $P<0.05$.

The number of embryos transferred per patient was one to three for day 3 and one to two for day 5 embryos transferred despite of that the multiple pregnancy rate was not significantly different between both groups.

An increase in miscarriage rate with day 5 embryo transferred compared with day 3 embryos $(12.0 \%$ vs. $4.4 \%, P=0.01)$, however no significant differences in biochemical pregnancy rate $(P=0.52)$.

\section{Discussion}

The advances in embryonic culture allow for extension of embryo growth to blastocyst stage. This prolongation of culture to day 5 may allow chromosomally competent embryos to develop to the blastocyst stage, thereby promoting intact embryos[2]. We believe that extension of embryo culture to day 5 will promote better quality of embryos for transfer, additionally, less number of embryos will be transferred so as to decrease the number of high order multiple pregnancies. In the other hand, this might allow for arrested embryo growth as some embryos will not withstand this extended period of culture and this can lead to cancellation of the treatment cycle. However, the results of previous randomized clinical trials and retrospective observations that examine day 3 compared to day 5 embryos transfer are variable and inconsistent[11].

Our study showed that both pregnancy and implantation rates were statistically insignificant between day 3 and day 5 , which was consistent with several previous studies. Coskun et al.[1], Levron et $a l .[14]$ did not find a difference in implantation and pregnancy rates of patients receiving day 3 and day 5 embryo transfer. While Karaki et al.[5] demonstrated that embryo culture to the blastocyst stage resulted in a significantly higher implantation rate than embryos transferred on day 3. Blastocyst transfer achieved an equivalent pregnancy rate with significantly fewer embryos than did on day 3 embryo transfer, as well as significantly fewer high order multiple gestations. Papanikolaou et al.[9] showed that the implant tation potential of an in vitro cultured blastocyst is higher compared with an in vitro cleavage-stage embryo. This meta-analysis supported this finding, when an equal number and up to two embryo transfer policy is followed with risk of higher probability of embryo transfer cancellation and less number of embryos cryopreserved in the blastocyst group[9]. This is considered a major drawback with possibility of failure to develop blastocysts stage embryo and thus failed to transfer embryos[15,16].

Most studies have shown unclear significance among high multiple pregnancy rate. While some studies reported lower rates of multiple pregnancy for blastocyst transfer, others have shown no difference[17-19] and increased multiple gestations in the day 3 groups[20,21] which was not seen in the former studies[11]. In our study, although the day 5 embryo transfer group had one or two embryos transferred compared with day 3 embryo transfer group who had three or less number of embryos transferred, there was no decrease in the multiple pregnancy rates in day 5 transfer group.

Our study data showed a higher miscarriage rate with day 5 embryo transfer $(12.03 \%)$ compared with day 3 embryo transfer $(4.40 \%)(P$ $<0.011)$.

This could be explained by two theories. Firstly, extension of embryo culture may allow for more stronger endometrium to develop result in defective implantation and increased miscarriage rate. Secondly, culture media may play a significant role. Studied in bovine demonstrated that change in implantation and placentation can cause miscarriages and that might be applied to human embryos in some way. Lonergan et al.[22], Tesfaye et al.[23] both studies suggest that the post-fertilization embryo culture environment in 
bovine has a dramatic effect on the pattern of gene expression in embryos, which in turn has serious implications for the normality of blastocyst development. Rizos et al.[24,25] showed that the type of media solution and in vitro culture itself can cause changes in bovine embryos. Changes in placental morphology have also been attributed to in vitro culture of bovine embryos. Decreased villous volume density and binucleate cell volume density were found in bovine embryos cultured in vitro when compared with in vivo embryos[26]. Utsunomiya et al.[27] showed a higher miscarriage rate on blastocyst transfer compared with day 3 embryo transfer and only the findings of this study were consisted with ours. However, a limitation existed in our study. Our study was not randomized and a potential bias in the methodology was present in spite of both groups studied were closely matched. All the data collected in the same period but a prospective randomized study could provide a definitive answer as to the usefulness of extended culture of embryos and associated risk of miscarriage. On other hand, our lab did not allow for extension of embryo, if the embryos showed evidence of slow growth. Early embryos transfer was done and this is another source of bias.

In conclusion, the presented data suggests that day 5 embryo transfers may not provide any additional benefits over day 3 transfers to patients. Also, it increases the risk of miscarriage. Further studies of this issue need to be undertaken to confirm our findings.

\section{Conflict of interest statement}

The authors declare that they have no conflicts of interest.

\section{References}

[1] Coskun S, Hollanders J, Al-Hassan S, Al-Sufyan H, Al-Mayman H, Jaroudi K. Day 5 versus day 3 embryo transfer: A controlled randomized trial. Hum reprod 2000; 15(9): 1947-1952.

[2] Schwarzler P, Zech H, Auer M, Pfau K, Gobel G, Vanderzwalmen P, et al. Pregnancy outcome after blastocyst transfer as compared to early cleavage stage embryo transfer. Hum Reprod 2004; 19(9): 2097-2102.

[3] Olivennes F, Hazout A, Lelaidier C, Freitas S, Fanchin R, de Zeiglar D, et al. Four indications for embryo transfer at the blastocyst stage. Hum Reprod 1994; 9(12): 2367-2373.

[4] Scholtes MCW, Zeilmaker GH. A prospective, randomized study of embryo transfer results after 3 or 5 days of embryo culture in in vitro fertilization. Fertil Steril 1996; 65(6): 1245-1248.

[5] Karaki R, Samarraie S, Younis N, Lahloub T, Ibrahim M. Blastocyst culture and transfer: A step toward improved in vitro fertilization outcome. Fertil Steril 2002; 77(1): 114-118.

[6] Jones GM, Trounson AO. Blastocyst stage transfer: Pitfalls and benefits (the benefits of extended culture). Hum Reprod 1999; 14(6): 1405-1408.

[7] Racowsky C, Jackson KV, Cekleniak NK, Fox JH, Hornstein MD, Ginsburg ES. The number of eight-cell embryos is a key determinant for selecting day 3 or day 5 transfer. Fertil Steril 2000; 73(3): 558-564.

[8] Lopta A. The neglected human blastocyst. J Assist Reprod Genet 1992; 9(6): 509-512.

[9] Papanikolaou1 E, Kolibianakis E, Tournaye H, Venetis C, Fatemi H, Tarlatzis B, et al. Live birth rates after transfer of equal number of blastocysts or cleavage-stage embryos in IVF. A systematic review and meta-analysis. Hum Reprod 2008; 23(1): 91-99.

[10]Milki A, Hinckley M, Fisch J, Dasig D, Behr B. Comparison of blastocyst transfer with day 3 embryo transfer in similar patient populations. Fertil
Steril 2000; 73(1): 126-129.

[11]Beesley R, Robinson R, Propst A, Arthur N, Retzloff M. Impact of day 3 or day 5 embryo transfer on pregnancy rates and multiple gestations. Fertil Steril 2009; 91(5): 1717-1720.

[12]Papanikolaou EG, D'haeseleer E, Verheyen G, Van de Velde H, Camus M, Steirteghem A, et al. Live birth rate is significantly higher after blastocyst transfer than after cleavage-stage embryo transfer when at least four embryos are available on day 3 of embryo culture. A randomized prospective study. Hum Reprod 2005; 20(11): 3198-3203.

[13]Freeman M, Howard K, Hinds M, Whitworth C, Weitzman G, Hill G. Embryo transfer : A retrospective comparison of day-5 blastocyst transfer versus day-3 embryo transfer. Fertil Steril 2000; 74(3): 170-171.

[14]Levron J, Shulman A, Bider D, Seidman D, Levin T, Dor J. A prospective randomized study comparing day 3 with blastocyst-stage embryo transfer. Fertil Steril 2002; 77(6): 1300-1301.

[15]Scholtes MCW, Zeilmaker GH. Blastocyst transfer in day-5 embryo transfer depends primarily on the number of oocytes retrieved and not on age. Fertil Steril 1998; 69(1): 78-83.

[16]Tsirigotis M. Blastocyst stage transfer: Pitfalls and benefits. Too soon to abandon current practice. Hum Reprod 1998; 13(12): 3285-3289.

[17]Rienzi L, Ubaldi F, Iacobelli M, Ferrero S, Minasi MG, Martinez F, et al. Day 3 embryo transfer with combined evaluation at the pronuclear and cleavage stages compares favorably with day 5 blastocyst transfer. Hum Reprod 2002; 17(7): 1852-1855.

[18]Coskun S, Hollanders J, Hassan S, Al-Sufyan H, Al-Mayman H, Jaroudi K. Day 5 versus day 3 embryo transfer: A controlled randomized trial. Hum Reprod 2000; 15(9): 1947-1952.

[19]Karaki RZ, Samarriae SS, Younis NA, Lahloub TM, Ibrahim MH. Blastocyst culture and transfer: A step toward improved in vitro fertilization outcome. Fertil Steril 2002; 77(1): 114-118.

[20]Estes SJ, Hoover LM, Smith SE, Somkuti SG, Schinfeld JS, Barmat LI. Comparsion of pregnancy, implantation, and multiple gestation rates for day 3 versus day 5 embryo transfers. J Assist Reprod Genet 2003; 20(10): 409-412.

[21]Toledo AA, Wright G, Jones AE, Smith SS, Johnson-Ward J, Brockman WW, et al. Blastocyst transfer: A useful tool for reduction of high-order multiple gestations in human assisted reproduction program. Am J Obstet Gynecol 2000; 183(2): 377-382.

[22]Lonergan P, Rizos D, Gutierrez-Adan A, Fair T, Boland MP. Oocyte and embryo quality: Effect of origin, culture conditions and gene expression patterns. Reprod Domest Anim 2003; 38(4): 259-267.

[23]Tesfaye D, Ponsuksili S, Wimmers K, Gilles M, Schellander K. A comparative expression analysis of gene transcripts in post-fertilization developmental stages of bovine embryos produced in vitro or in vivo. Reprod Domest Anim 2003; 39(6): 396-404.

[24]Rizos D, Lonergan P, Ward F, Duffy P, Boland MP. Consequences of bovine oocyte maturation, fertilization or early embryo development in vitro versus in vivo: Implications for blastocyst yield and blastocyst quality. Molr Reprod Dev 2002; 61(2): 234-248.

[25]Rizos D, Fair T, Papadopoulos S, Boland MP, Lonergan P. Developmental, qualitative, and ultrastructural differences between ovine and bovine embryos produced in vivo or in vitro. Mol Reprod Dev 2002; 62(3): 320-327.

[26]Dar S, Librach CL, Gunby J, Bissonnette F, Cowan L. Increased risk of preterm birth in singleton pregnancies after blastocyst versus day 3 embryo transfer: Canadian ART register (CARTR) analysis. Hum Reprod 2013; 28(4): 924-928.

[27]Takafumi Utsunomiya, Hiroko Ito, Miyuki Nagaki, Junko Sato. A prospective, randomized study: Day 3 versus hatching blastocyst stage. Human Reproduction 2004; 19(7): 1598-1603. 